\title{
CD30-Positive T-Cell Lymphoproliferative Disease of the Oral Mucosa in Children: A Manifestation of Epstein-Barr Virus-Associated T-Lymphoproliferative Disorder
}

\author{
Mineui Hong · Young Hyeh Ko ${ }^{1}$ \\ Department of Pathology, Hallym University \\ Kangnam Sacred Heart Hospital, \\ Hallym University College of Medicine, Seoul; \\ 'Department of Pathology, \\ Samsung Medical Center, \\ Sungkyunkwan University School of Medicine, \\ Seoul, Korea \\ Received: May 14, 2015 \\ Revised: July 10, 2015 \\ Accepted: July 13, 2015 \\ Corresponding Author \\ Young Hyeh Ko, MD, PhD \\ Department of Pathology, Samsung Medical Center, \\ Sungkyunkwan University School of Medicine, \\ 81 Irwon-ro, Gangnam-gu, Seoul 06351, Korea \\ Tel: +82-2-3410-2762 \\ Fax: +82-2-3410-0025 \\ E-mail: yhk0310@skku.edu
}

\begin{abstract}
Eosinophilic ulcer of the oral mucosa (EUOM) is a very rare, benign, self-limiting ulcerative lesion of the oral cavity of unknown pathogenesis, and belongs to the same spectrum of CD30+ T-cell lymphoproliferative disease (LPD) of the oral mucosa. The etiology and pathogenesis of the disease are unknown. We report two cases in children who were initially diagnosed with EUOM and $\mathrm{CD} 30^{+} \mathrm{T}$-cell LPD, respectively. However, retrospective analysis revealed that a majority of infiltrated atypical T cells were positive for Epstein-Barr virus (EBV). The present cases suggest that the pathogenesis and etiology of EUOM or CD30+ T-cell LPD occurring in children are different from those in adults. EUOM or CD30+ T-cell LPD in children is a manifestation of EBV-positive T-cell LPD, and should therefore be distinguished from the disease in adults.
\end{abstract}

Key Words: Eosinophilic ulcer; CD30 positive; Lymphoproliferative disorders; Oral mucosa; EpsteinBarr virus infections; Lymphoreticular
$\mathrm{CD} 30^{+}$T-cell lymphoproliferative disease (LPD) of the oral mucosa is uncommon and belongs to the spectrum of mucosal CD $30^{+}$T-cell LPD, which share pathological and clinical features as follows. ${ }^{1}$ CD $30^{+}$T-cell LPD is an indolent disease and often spontaneously regresses and never appears to progress to systemic disease. The lesion can develop in the gingiva, buccal mucosa, palate, or tongue. It comprises dense infiltrates of $\mathrm{CD} 30^{+}$ atypical T-cells with polymorphous inflammatory infiltrate in the background, which frequently includes eosinophils. In a number of previous investigations, atypical T-cells were $\mathrm{CD}^{+}$ $\mathrm{CD}^{+}{ }^{+} \mathrm{CD}_{6} 6^{-}$cytotoxic molecules ${ }^{+}$such as TIA. T-cell receptor (TCR) gene rearrangement is clonal in the majority of cases, ${ }^{2}$ but in some cases it is polyclonal. ${ }^{3,4}$ Eosinophilic ulcer of the oral mucosa (EUOM) which is one of the differential diagnoses of CD $30^{+}$T-cell LPD is a very rare, benign, self-limited, ulcerative lesion of the oral cavity of unknown pathogenesis. EUOM has been reported under various synonyms, including traumatic ulcerative granuloma with stromal eosinophilia, traumatic granuloma, traumatic eosinophilic granuloma, and eosinophilic gran- uloma of the tongue. ${ }^{5-10}$ EUOM occurs mainly in adults, but shows two age peaks: during the first two years of life and between the sixth and seventh decades. ${ }^{11}$ The etiology of EUOM is unknown. Several reports proposed that some viral or toxic agents may play a role in the development of EUOM or that trauma is a contributing factor. ${ }^{5}$ Evidence that has accumulated mostly over the past decade suggests that EUOM and CD $30^{+} \mathrm{T}$ cell LPD are closely related and that EUOM can be included within the spectrum of $\mathrm{CD} 30^{+}$T-cell LPD. ${ }^{1,26-9,11}$ In addition, the Epstein-Barr virus (EBV) status of these diseases has not been defined yet. Herein, we report two cases of CD $30^{+}$T-cell LPD or EUOM in children, which revealed clonal proliferation of T-cells harboring EBV.

\section{CASE REPORT}

Case 1

A 13-year-old boy was referred to our hospital because of a twoweek history of a painful $4 \mathrm{~cm}$ ulcer on his left lower gingiva. The 
sharply demarcated ulcer developed spontaneously without mechanical irritation. Before admission, he had suffered from recurrent stomatitis and had high fever, night sweats, and diarrhea. He was treated with antibiotics and an antipyretic, but the ill- ness was persistent. On physical examination, he appeared acutely ill. The ulcer along the buccal gingiva was linear with a sharp erythematous margin, and was covered with a purulent exudate (Fig. 1A). He was diagnosed with EUOM before the referral, and
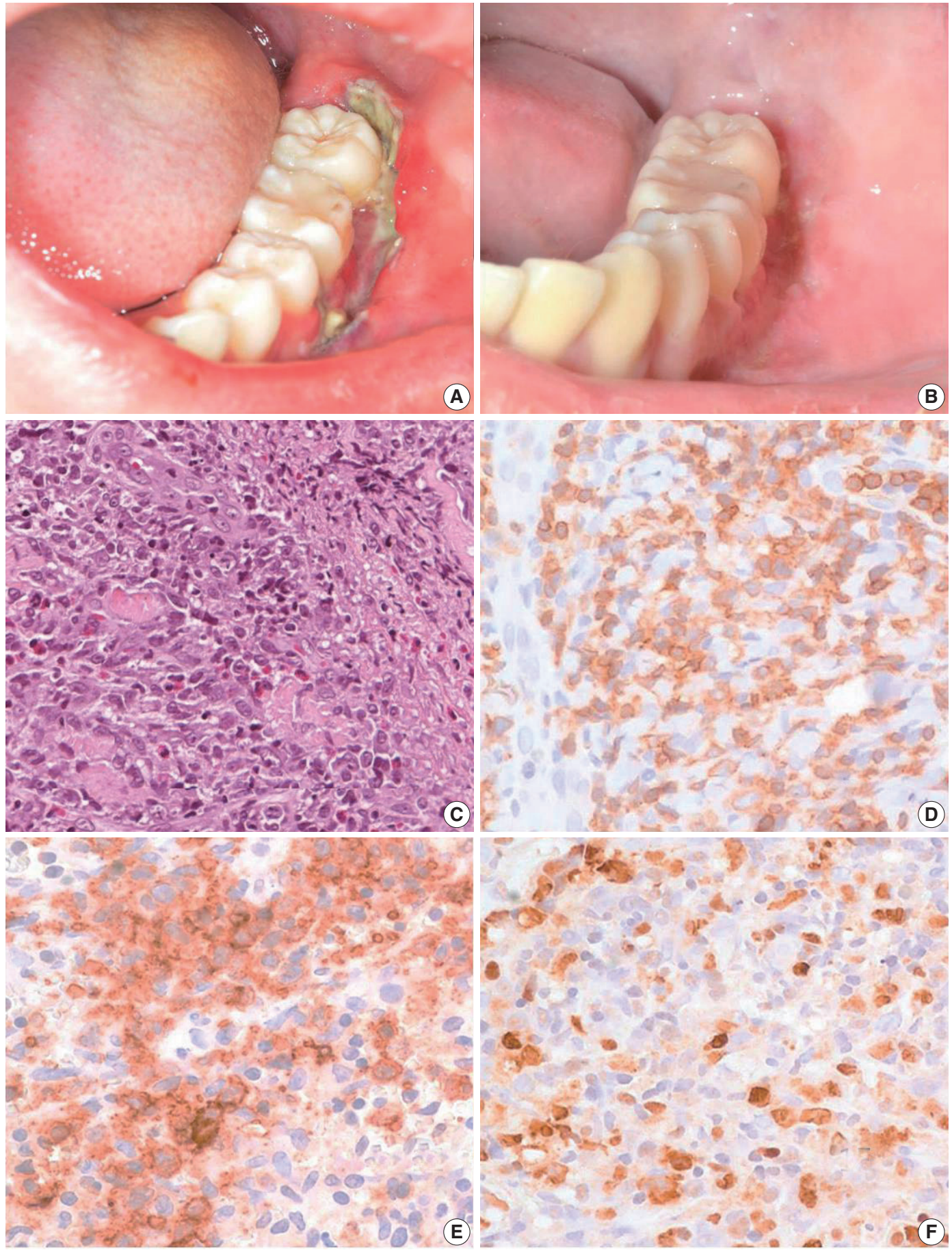

Fig. 1. Clinical finding and histopathologic findings of the oral lesion in case 1. A sharply demarcated linear ulcer was seen at the left lower gingiva (A), which was completely healed 10 days after diagnosis (B). (C) Histologically, infiltration of large atypical cells with many eosinophils was found. (D) CD3 staining highlights large T-blasts. These cells are also stained with CD30 (E) and Epstein-Barr virus (EBV) by EBV-encoded RNA in situ hybridization (F). 
the pathology slide was reviewed by the authors.

On retrospective review, biopsy revealed diffuse infiltration of large atypical $\mathrm{CD}^{+}{ }^{+} \mathrm{T}$-cells intermixed with numerous eosinophils and small lymphocytes in the submucosa and soft tissue with necrosis. These cells were $\mathrm{CD}^{+}, \mathrm{CD} 0^{+}$, and $\mathrm{CD} 56$. Some small or large lymphocytes were positive for CD4, TCR $\beta \mathrm{F} 1$, and granzyme B. EBV-encoded RNA (EBER) was detected in many large atypical cells by in situ hybridization (Fig. 1C-F). TCR $\gamma$ gene rearrangement study revealed monoclonality (Fig. 2A). Epstein-Barr (EB) viral load in blood was elevated. The diagnosis was revised to EBV-positive T-cell LPD. He was treated with chemotherapy with 106B induction (prednisolone, cyclophosphamide, daunorubicin, vincristine, and L-asparaginase). After 10 days from the diagnosis, the ulcer was resolved. During the 21 months of follow-up period, the oral ulcer showed a wax and wane pattern without further treatment. The patient was quite well; however, EB viral load was persistently high with 34.5 copies $/ \mu \mathrm{L}$ in whole blood (normal range up to 1.02 copies $/ \mu \mathrm{L}$ ) at the last visit (Fig. 2B).

\section{Case 2}

An 11-year-old girl was admitted with a 20-day history of buccal abscess. She had suffered from frequent infections since infancy, such as pneumonia, bronchiolitis, and acute gastroenteritis. She also suffered from a two-year history of recurrent fever and oral ulcer. The oral ulcer would develop every two months and spontaneously regress. Two years earlier, a biopsy of the oral ulcer was conducted at another hospital, and was diagnosed as chronic inflammation. Before admission, she showed a buccal abscess and submandibular lymphadenopathy. At admission, there was a $0.3 \mathrm{~cm}$ oral ulcer on her left inferior gingiva and broad ulcerative lesion on her palate. Complete blood count was within normal range with a normal T-cell subset. Blood chemistry was within normal limits except for a slightly increased alkaline phosphatase (125 U/L, normal range 42-98 U/L). T lymphocyte proliferative activity in response to mitogens (phytohemagglutinin and concanavalin A) was preserved. A nitrobluetetrazolium test to exclude chronic granulomatous disease was negative. A biopsy from the buccal ulcer showed mucosal ulcer-
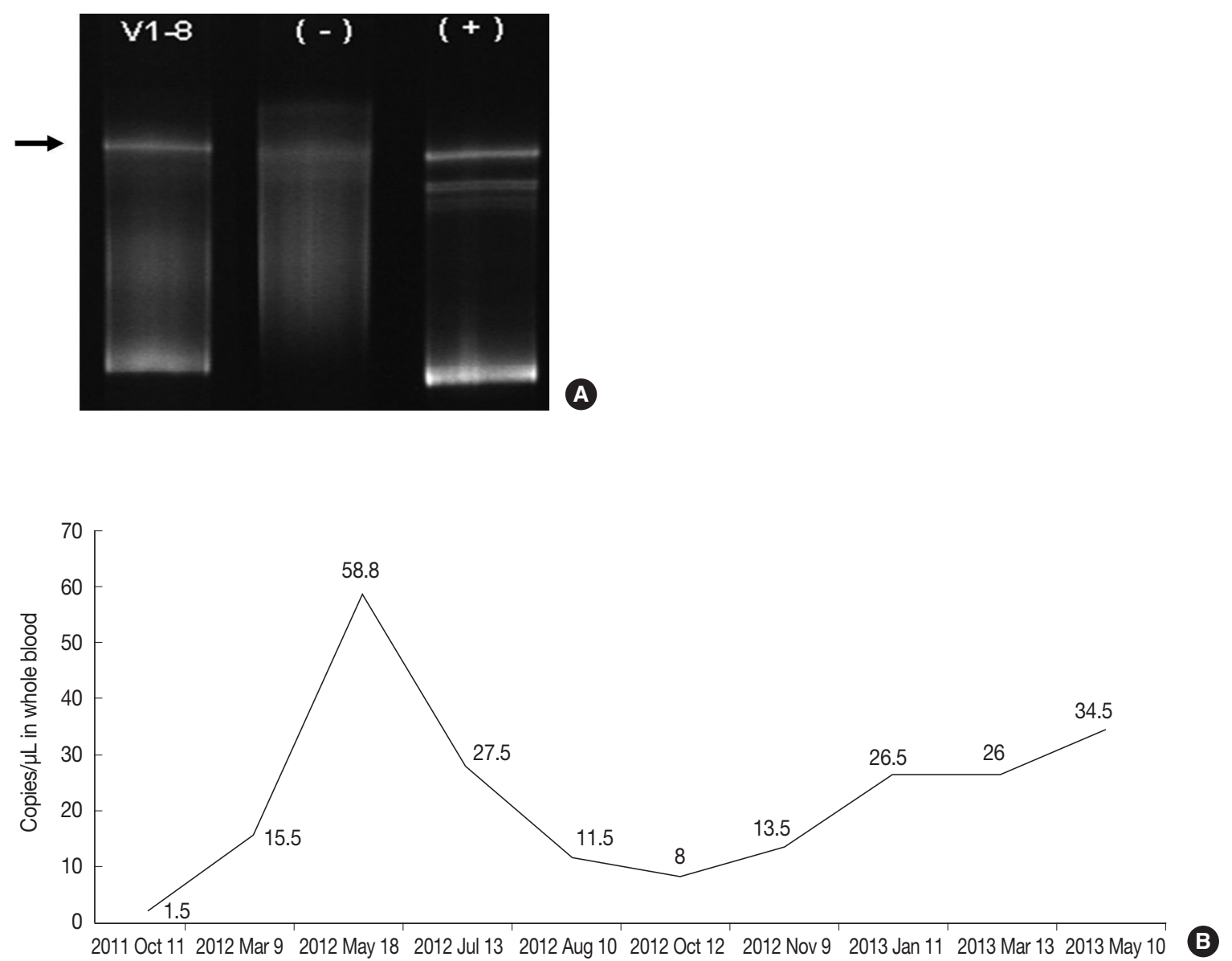

Fig. 2. (A) TCR $\gamma$ gene rearrangement in case 1 showed a monoclonal band. (B) Epstein-Barr viral load analyzed in blood was persistently elevated in case 1 up to 58.8 copies/ $\mu \mathrm{L}$ for two years until last follow up. 




Fig. 3. Histopathologic findings of oral lesion in case 2. (A) Large cells are scattered among small lymphocytes. (B) These cells are positive for CD3. (C) CD30 were positive in the cytoplasmic membrane and perinuclear zone of large atypical lymphocytes. (D) Large cells are also positive for Epstein-Barr virus-encoded RNA in situ hybridization.

ation with marked inflammatory cell infiltration including eosinophils. The ulcerated mucosa was covered by fibrinopurulent exudate. The submucosa and adjacent skeletal muscle showed diffuse or patchy infiltration of mixed small, medium, and large lymphocytes (Fig. 3A). These lymphocytes were $\mathrm{CD}^{+}$, which highlighted the irregular nuclear contour of atypical T cells (Fig. 3B). CD30 was positive in the cytoplasmic membrane and perinuclear zone of large atypical lymphocytes (Fig. 3C). Anaplastic lymphoma kinase (ALK) protein was negative. Under the diagnosis of $\mathrm{CD} 30^{+} \mathrm{T}$-cell LPD, a subset of ALK-negative anaplastic large cell lymphoma, the patient was treated with chemotherapy with methotrexate and cyclophosphamide. After 15 months of chemotherapy, the patient was stable without disease progression. However, on retrospective review of the specimen, the diagnosis was revised as EBV-positive T-cell LPD, most likely chronic active EBV (CAEBV) infection because of EBV positivity in the majority of the small to large atypical cells (Fig. 3D).

\section{DISCUSSION}

One of the main differential diagnoses of $\mathrm{CD} 30^{+} \mathrm{T}$-LPD of the oral cavity is EUOM. EUOM is characterized by mucosal ulceration with an underlying extensive inflammatory infiltrate consisting of $\mathrm{B}$ and $\mathrm{T}$ lymphocytes, macrophages, abundant eosinophils, and large atypical mononuclear cells of variable proportion. Atypical lymphoid infiltration and CD30 positivity can be seen to some extent, in $41 \%$ to $70 \%$ of cases. ${ }^{1,6-8,10}$ Monoclonal T-cell proliferation was reported in 12 of 48 cases analyzed. ${ }^{1,7,810}$ However, despite the presence of $\mathrm{CD} 30$ expression or T-cell monoclonality, the prognosis of EUOM is known to be excellent. Based on the similarity of clinicopathologic features between EUOM and mucosal CD30 ${ }^{+}$T-cell LPD, at least a subset of EUOM might be CD30 ${ }^{+}$T-cell LPD of the oral mucosa. In EUOM or $\mathrm{CD} 30^{+} \mathrm{T}$-cell LPD, EBV status was unknown because it is usually not examined in routine diagnosis. So far, there are only two reports of EBV status. Agarwal et al. ${ }^{1}$ examined three adult patients with oral CD30 ${ }^{+}$T-cell LPD by EBER in situ hybridization and reported no association between $\mathrm{EBV}$ and $\mathrm{CD} 30^{+}$ T-cell LPD. Abdel-Naser et al. ${ }^{11}$ first demonstrated the association between EBV and EUOM in a 12-year-old boy with an eosinophilic oral ulcer. EBV latent membrane protein was expressed in coexistence with $\mathrm{CD} 30^{+} \mathrm{T}$ lymphocytes. Epstein-Barr nucle- 
ar antigen 2 or Zebra antigen was negative. Gene rearrangement studies revealed polyclonal B cells and oligoclonal T cells. ${ }^{11}$ Notably, the patient reported by Abdel-Naser et al. ${ }^{11}$ and our two patients were children. After primary infection of EBV, most children will completely recover; however, some children can be rarely complicated by severe or persistent infections such as acute hemophagocytic lymphohistiocytosis, systemic T-cell LPD, or CAEBV infection. ${ }^{12-14}$ CAEBV infection mainly involves $\mathrm{T}$ or natural killer (NK) cells and is accompanied by varying degrees of lymphoproliferation, which may progress from polyclonal lymphoproliferation to monoclonal disease. ${ }^{15}$ Usually, systemic symptoms such as fever persist for more than six months with increased EB viral load in blood or tissues. Although the most frequent symptoms include organomegaly, anemia, and thrombocytopenia, patients often present with uncommon clinical findings such as coronary aneurysms, central nervous system involvement, bowel perforation, or Behçet-like orogenital ulcer. ${ }^{16}$ The clinical course is variable and depends on the immune response of the individuals. Some patients have a prolonged and indolent disease course, whereas half the patients die of hemophagocytic syndrome or aggressive NK cell leukemia/lymphoma. ${ }^{15}$ One of our patients showed persistently elevated EB viral load in the blood and infiltration of clonal EBV-positive T-cells in an oral ulcerative lesion. We did not examine EB viral load in the blood of the case 2 because this was a retrospective study. Persistent elevation of EB viral load in the blood of the case 1 with monoclonality of infiltrated $\mathrm{CD} 30^{+}$cells suggest that the oral ulcer of these two patients is a manifestation of EBV-associated T-cell LPD, which is clinically consistent with an indolent form of CAEBV infection.

An important differential diagnosis is EBV-positive mucocutaneous ulcer, which is part of the spectrum of age-related EBVpositive LPD. According to Dojcinov et al., ${ }^{17}$ the median age of patients was 77 years. In addition, EBV-infected large blasts were mainly B-cells with CD20 expression. The present cases are distinct from EBV-positive mucocutaneous ulcer in their EBV positivity in T-cells and the young age of the patients.

In conclusion, our cases suggest that the pathogenesis and etiology of so-called "oral eosinophilic ulcer" or CD $30^{+}$T-cell LPD occurring in children are different from the disease in adults. EUOM and CD $30^{+}$T-cell LPD in children is a manifestation of EBV-positive T-cell LPD, and therefore should be distinguished from those of adults. Examination of EBV status in more cases of children and adult patients would provide more convincing data to support our observations.

\section{Conflicts of Interest}

No potential conflict of interest relevant to this article was reported.

\section{REFERENCES}

1. Agarwal M, Shenjere P, Blewitt RW, et al. CD30-positive T-cell lymphoproliferative disorder of the oral mucosa: an indolent lesion: report of 4 cases. Int J Surg Pathol 2008; 16: 286-90.

2. Sciallis AP, Law ME, Inwards DJ, et al. Mucosal CD30-positive Tcell lymphoproliferations of the head and neck show a clinicopathologic spectrum similar to cutaneous CD30-positive T-cell lymphoproliferative disorders. Mod Pathol 2012; 25: 983-92.

3. Wang W, Cai Y, Sheng W, Lu H, Li X. The spectrum of primary mucosal CD30-positive T-cell lymphoproliferative disorders of the head and neck. Oral Surg Oral Med Oral Pathol Oral Radiol 2014; 117: 96-104.

4. Erõs N, Marschalkó M, Lõrincz A, et al. CD30-positive anaplastic large T-cell lymphoma of the tongue. J Eur Acad Dermatol Venereol 2009; 23: 231-2.

5. Tang TT, Glicklich M, Hodach AE, Oechler HW, McCreadie SR. Ulcerative eosinophilic granuloma of the tongue: a light- and electron-microscopic study. Am J Clin Pathol 1981; 75: 420-5.

6. Ficarra G, Prignano F, Romagnoli P. Traumatic eosinophilic granuloma of the oral mucosa: a CD30+(Ki-1) lymphoproliferative disorder? Oral Oncol 1997; 33: 375-9.

7. Alobeid B, Pan LX, Milligan L, Budel L, Frizzera G. Eosinophil-rich CD30+ lymphoproliferative disorder of the oral mucosa: a form of "traumatic eosinophilic granuloma". Am J Clin Pathol 2004; 121: $43-50$.

8. Salisbury CL, Budnick SD, Li S. T-cell receptor gene rearrangement and CD30 immunoreactivity in traumatic ulcerative granuloma with stromal eosinophilia of the oral cavity. Am J Clin Pathol 2009; 132: $722-7$.

9. Segura S, Romero D, Mascaró JM Jr, Colomo L, Ferrando J, Estrach T. Eosinophilic ulcer of the oral mucosa: another histological simulator of CD30+ lymphoproliferative disorders. Br J Dermatol 2006; 155: 460-3.

10. Hirshberg A, Amariglio N, Akrish S, et al. Traumatic ulcerative granuloma with stromal eosinophilia: a reactive lesion of the oral mucosa. Am J Clin Pathol 2006; 126: 522-9.

11. Abdel-Naser MB, Tsatsou F, Hippe S, et al. Oral eosinophilic ulcer, an Epstein-Barr virus-associated CD30+ lymphoproliferation? Dermatology 2011; 222: 113-8.

12. Cho EY, Kim KH, Kim WS, Yoo KH, Koo HH, Ko YH. The spec- 
trum of Epstein-Barr virus-associated lymphoproliferative disease in Korea: incidence of disease entities by age groups. J Korean Med Sci 2008; 23: 185-92.

13. Wang RC, Chang ST, Hsieh YC, et al. Spectrum of Epstein-Barr virus-associated T-cell lymphoproliferative disorder in adolescents and young adults in Taiwan. Int J Clin Exp Pathol 2014; 7: 2430-7.

14. Park S, Ko YH. Epstein-Barr virus-associated T/natural killer-cell lymphoproliferative disorders. J Dermatol 2014; 41: 29-39.

15. Ohshima K, Kimura H, Yoshino T, et al. Proposed categorization of pathological states of EBV-associated T/natural killer-cell lympho- proliferative disorder (LPD) in children and young adults: overlap with chronic active EBV infection and infantile fulminant EBV TLPD. Pathol Int 2008; 58: 209-17.

16. Park BM, Ahn JS, Lee JB, Won YH, Yun SJ. Chronic active EpsteinBarr virus infection-associated hydroa vacciniforme-like eruption and Behcet's-like orogenital ulcers. Dermatology 2013; 226: 212-6.

17. Dojcinov SD, Venkataraman G, Raffeld M, Pittaluga S, Jaffe ES. EBV positive mucocutaneous ulcer: a study of 26 cases associated with various sources of immunosuppression. Am J Surg Pathol 2010; 34: 405-17. 